\title{
Estudio de seguimiento a cinco años de la psicosis aguda: evolución diagnóstica y adaptación psicosocial.
}

Five years follow-up study of the acute psychosis: diagnostic evolution and psychosocial adjustment.

\author{
A. Pedrós Roselló a , J. Ma Tenías Burillob. \\ ${ }^{a}$ Servicio de Psiquiatría, Xátiva, España. ${ }^{b}$ Unidad de Apoyo a la Investigación del Hospital General Man- \\ cha Centro, Alcázar de San Juan, España.
}

Correspondencia: A. Pedrós Roselló (pedros_alf@gva.es)

Recibido: 30/06/2011; aceptado con modificaciones: 19/10/2011

\begin{abstract}
RESUMEN: Introducción. Existe un grupo de psicosis agudas que presenta un pronóstico más favorable. Sin embargo, algunas de estas psicosis pueden evolucionar hacia enfermedades crónicas psicóticas o afectivas. El objetivo de este trabajo es el estudio de la evolución diagnóstica y pronóstico en psicosis aguda.

Método. Estudio observacional durante cinco años de una muestra poblacional de pacientes con psicosis aguda.

Resultados. A los cinco años el $73.2 \%$ de los 41 pacientes analizados recibe un diagnóstico de enfermedad psicótica o afectiva crónica, presentando mayor deterioro en su adaptación y calidad de vida. Aproximadamente un $50 \%$ de los casos con psicosis breve al inicio del estudio continúa manteniendo dicho diagnóstico. Aunque no existen diferencias en la evolución diagnóstica en relación al género, las mujeres presentan un menor grado de deterioro con una mejor adaptación.

Conclusiones. A los cinco años la mayoría de los pacientes han desarrollado una enfermedad psicótica o afectiva crónica. El único diagnóstico con mayor estabilidad y mejor pronóstico es el de trastorno psicótico breve, sobre todo con acontecimientos vitales desencadenantes. Las mujeres presentan un menor deterioro y mejor adaptación y calidad de vida que los hombres. PALABRAS CLAVE: Psicosis aguda. Adaptación social. Calidad de vida. Estudios de seguimiento.
\end{abstract}

ABSTRACT: Introduction. A group of acute psychosis presents a more favourable prognosis. However, some of these can develop into chronic psychotic or affective disorders. The objective is the study of diagnostic stability and prognosis in acute psychosis.

Method. Five years follow-up study of acute psychosis in a population sample.

Results. Within five years, $73.2 \%$ of the 41 patients studied received a diagnosis of chronic psychotic or affective illness, presenting further deterioration in their adaptation and quality on life. Approximately $50 \%$ of cases with brief psychosis at baseline maintained that diagnosis. Although there were no differences in the diagnoses with respect to gender, women exhibited a lesser degree of degradation along with better adjustment.

Conclusions. The majority of patients diagnosed developed a chronic psychotic or affective illness during the five year period. The only diagnosis with a greater stability and better prognosis was that of brief psychosis disorder, especially triggered with life events. Women exhibited a better evolution and ability to adapt than men.

KEY WORDS: Acute psychosis. Social adjustment. Quality of life. Follow up studies.

\section{Introducción}

Existen estudios que apoyan la existencia de un grupo de psicosis agudas que suponen un mejor pronóstico. Se habla de psicosis con un inicio agudo (menos de dos semanas), sintomatología dramática y polimorfa y una rápida resolución (1). Estas psicosis agudas o transitorias difieren de diagnósticos más estables como es- 
quizofrenia, esquizoafectivo o bipolar, observándose una menor especificidad (2). A través de trabajos de seguimiento se ha observado que estos episodios psicóticos agudos pueden ser el inicio de un posterior diagnóstico de trastorno psicótico o afectivo de evolución crónica. En estos casos es la evolución en el tiempo, en ocasiones se habla de cinco años, la que establece un diagnóstico más definitivo. El análisis de la estabilidad diagnóstica de la psicosis aguda es de crucial importancia para el pronóstico (3-6). Por este motivo, resulta de especial importancia poder identificar variables o factores que puedan contribuir a un diagnóstico futuro más favorable.

El objetivo de este trabajo de investigación es analizar la evolución diagnóstica y funcionamiento a los cinco años de pacientes diagnosticados al inicio de psicosis aguda e identificar factores que puedan influir en el pronóstico.

\section{Método.}

\section{Muestra.}

En un estudio previo se analizó el diagnóstico a los dos años de 48 pacientes inicialmente diagnosticados de psicosis aguda (trastorno psicótico breve, trastorno psicótico no especificado, trastorno psicótico inducido por drogas). Los episodios psicóticos deben cumplir además los siguientes criterios: inicio agudo (menor a cuatro semanas), con o sin factores desencadenantes y sin haber recibido tratamiento neuroléptico previo al ingreso. Los criterios de exclusión son diagnóstico previo de psicosis crónica y sospecha de origen orgánico del episodio actual. Asimismo, se estudia un grupo de pacientes con trastorno esquizofreniforme que precisan de ingreso durante el mismo período (7).

En este trabajo se presentan los resultados del estudio a los cinco años de seguimiento. Se contacta por teléfono o correo con el paciente y se concierta una entrevista en el servicio de Psiquiatría del H. Lluís Alcanyís de Xátiva (Valencia). Se realiza una valoración mental y diagnóstico psiquiátrico de los pacientes y un estudio de su funcionamiento psicosocial durante estos años. La muestra final para este seguimiento es de 41 pacientes $(n=41)$, tras 7 pérdidas por abandono o rechazo, 28 hombres $(68,3 \%)$ y 13 mujeres $(31,7 \%)$, con una edad media de 32.8 años (DE: 8,6 años, rango de 22 a 60 años).

\section{Procedimiento.}

La valoración del estado mental se realiza mediante entrevista clínica abierta, aplicación de la MINI Entrevista Neuropsiquiátrica Internacional (8) y la Escala para el Síndrome Positivo y Negativo de la Esquizofrenia (PANSS) (9). El diagnóstico definitivo se realiza con criterios DSM-IV (10), realizándose un agrupamiento 
ORIGINALES Y REVISIONES

sindrómico para el análisis estadístico (psicosis crónica, afectivo crónico y psicosis aguda).

Para el estudio del funcionamiento del paciente se abordan distintos aspectos como: funcionamiento general (escala heteroaplicada OMS-DAS: total, cuidado personal, ocupacional, familia y social) (11), adaptación social (escala autoaplicada SASS) (12) y calidad de vida (cuestionario autoaplicado CSCV) (13).

\section{Análisis estadístico.}

Se lleva a cabo un análisis descriptivo de las variables cuantitativas, mediante medidas de tendencia central (medias) y de dispersión (desviación estándar) y cualitativas, a través de frecuencias absolutas y relativas, expresadas como porcentajes.

Posteriormente, se realiza un análisis bivariante mediante contrastes de las escalas en relación a diferentes variables de agrupación (género, grupo diagnóstico), debido al bajo número de efectivos se utiliza la $U$ de Mann-Whitney para contrastes entre dos grupos (género) y la prueba de Kruskal-Wallis para grupos de más de dos categorías (diagnóstico). Finalmente, se llevan a término correlaciones entre escalas y otras variables numéricas (edad), se estiman los coeficientes de correlación no paramétricos de Spearman (Rho) al no poder asumir la normalidad ni la linealidad de las correlaciones.

Se utilizó como nivel de significación una $\mathrm{p}<0.05$. Todos los cálculos se realizaron con el paquete estadístico SPPS (versión 12.0).

Resultados.

Estabilidad y evolución del diagnóstico de psicosis aguda a los cinco años.

A los cinco años un $73.2 \%$ de los pacientes recibe un diagnóstico de proceso psicótico o afectivo crónico, mientras que un $26.8 \%$ continúa manteniendo el diagnóstico de psicosis aguda inicial. En esta evolución se debe destacar el cambio a esquizofrenia (34.2\%), seguido de esquizoafectivo (19.5\%) (tabla 1). Al analizar la evolución de los diagnósticos de forma individualizada se observa que los pacientes diagnosticados como esquizofreniforme evolucionan en su mayoría a esquizofrenia. Frente a ello, más de la mitad de pacientes con diagnóstico inicial de psicosis breve mantiene dicho diagnóstico (remitido) a los cinco años (tabla 2).

\section{Variables sociodemográficas y diagnóstico a los cinco años.}

Se analizan tres variables que pueden tener influencia en la evolución del diagnóstico (edad, género y acontecimientos vitales previos al inicio de la psicosis). La edad al inicio del episodio psicótico agudo no parece influir en el diagnóstico a los cinco años. En los hombres se observa, una mayor tendencia a sufrir 
una enfermedad psicótica crónica (en su mayoría esquizofrenia y esquizoafectivo), aunque sin significación estadística $(\mathrm{p}=0.11)$. La existencia de acontecimientos vitales en la precipitación del episodio psicótico agudo inicial se asocia con el mantenimiento de un diagnóstico de psicosis aguda (remitida) a los cinco años, mientras que la mayoría de los pacientes sin acontecimientos vitales desencadenantes $(84.6 \%)$ evolucionan hacia esquizofrenia o esquizoafectivo $(\mathrm{p}=0.08)($ tabla 3$)$.

Tabla 1.

Diagnóstico individual a los 5 años.

\begin{tabular}{|ll|}
\hline Diagnóstico DSM-IV & $\mathbf{N}(\boldsymbol{\%})$ \\
\hline Tno. esquizofrénico desorganizado & $1(2,4 \%)$ \\
Tno. esquizofrénico paranoide & $8(19,5 \%)$ \\
Tno. esquizofrénico residual & $3(7,4 \%)$ \\
Tno. esquizofrénico indiferenciado & $2(4,9 \%)$ \\
Tno. esquizofreniforme & $2(4,9 \%)$ \\
Tno. esquizoafectivo & $8(19,5 \%)$ \\
Tno. bipolar & $4(9,8 \%)$ \\
Tno. delirante & $1(2,4 \%)$ \\
Tno. psicótico breve & $7(17,1 \%)$ \\
Tno. psicótico no especificado & $4(9,8 \%)$ \\
Tno. depresivo no especificado & $1(2,4 \%)$ \\
\hline
\end{tabular}

Tabla 2.

Evolución diagnóstica a los 5 años.

\begin{tabular}{|l|c|c|c|c|}
\hline \multicolumn{1}{|c|}{ Dx final $\rightarrow$} & Psicosis crónica & Afectivo crónico & Psicosis Aguda & Total \\
\hline Tnx inicial & & & & 13 \\
\hline Tno. psquizofreniforme & $13(100 \%)$ & 0 & 0 & 6 \\
\hline Tno. psicótico inducido por drogas & $3(50,0 \%)$ & $1(16,7 \%)$ & $2(33,3 \%)$ & 13 \\
\hline Tno. psicótico no especificado & $5(38,5 \%)$ & $1(7,7 \%)$ & $7(53,8 \%)$ & 9 \\
\hline Total & $4(44,4 \%)$ & $3(33,3 \%)$ & $2(22,2 \%)$ & 41 \\
\hline
\end{tabular}

$\mathrm{P}=0,008$ (Ji cuadrado)

Tabla 3.

Variables sociodemográficas y diagnóstico a los 5 años.

\begin{tabular}{|l|c|c|c|c|}
\hline Variable & $\begin{array}{c}\text { Psicosis crónica } \\
(\mathbf{n = 2 5})\end{array}$ & $\begin{array}{c}\text { Afectivo crónico } \\
(\mathbf{n = 5})\end{array}$ & $\begin{array}{c}\text { Psicosis Aguda } \\
(\mathbf{n = 1 1})\end{array}$ & $\mathbf{p}^{*}$ \\
\hline Edad & $26,3(6,5)$ & $29,6(13,6)$ & $30,5(10,3)$ & 0,38 \\
Género & $5(20 \%)$ & $2(40 \%)$ & $6(54,5 \%)$ & 0,11 \\
$\quad$ Mujeres (13) & $20(80 \%)$ & $3(60 \%)$ & $5(45,5 \%)$ & \\
$\quad$ Hombres (28) & $11(84,6 \%)$ & 0 & $2(15,4 \%)$ & \\
Acontecimientos vitales & $14(50 \%)$ & $5(17,9 \%)$ & $9(32,1 \%)$ & \\
$\quad$ No (13) & & & \\
$\quad$ Sí (28) & & & \\
\hline
\end{tabular}

Resultados expresados como n (\%), excepto edad: Media (DE)

* Ji cuadrado; Edad: ANOVA 


\section{Diagnóstico, sintomatología psicótica y funcionamiento del paciente (adaptación social, discapacidad y calidad de vida) a los cinco años.}

Los resultados obtenidos muestran una coherencia interna y significación estadística, llegando a determinar una evolución y funcionamiento diferente para cada grupo de diagnóstico. La sintomatología psicótica positiva y negativa en los pacientes con proceso psicótico o afectivo crónico y los diagnosticados de psicosis aguda presentan diferencias estadísticamente significativas, sobre todo cuando se analiza el síndrome negativo $(\mathrm{p}<0.001)$. Al estudiar el funcionamiento de los pacientes, se obtienen tres niveles de adaptación/satisfacción claramente delimitados. Los pacientes con diagnóstico de psicosis crónica, presentan un mayor nivel de discapacidad (personal, ocupacional, familiar y social), un nivel de adaptación social menor y un nivel de calidad de vida más desfavorable que los pacientes con diagnóstico de bipolar. Los pacientes que a los cinco años mantienen un diagnóstico de psicosis aguda (remitida) muestran el mejor nivel de adaptación social, con un menor grado de discapacidad y una valoración más favorable de su calidad de vida. Todas estas asociaciones muestran un marcado nivel de significación estadística (tabla 4).

Tabla 4.

Diagnóstico a los 5 años y puntuaciones en las escalas PANSS, SASS, OMS-DAS y CSCV.

\begin{tabular}{|l|c|c|c|c|c|}
\hline Escala & $\begin{array}{c}\text { Global } \\
(\mathbf{n = 4 1})\end{array}$ & $\begin{array}{c}\text { Psicosis crónica } \\
(\mathbf{n = 2 5})\end{array}$ & $\begin{array}{c}\text { Afectivo crónico } \\
(\mathbf{n = 5})\end{array}$ & $\begin{array}{c}\text { Psicosis Aguda } \\
(\mathbf{n = 1 1})\end{array}$ & $\mathbf{p}^{*}$ \\
\hline PANSS-Positiva & $15,5(11,2)$ & $18,8(12,5)$ & $14,4(10,3)$ & $8,2(1,6)$ & 0,01 \\
PANSS-Negativa & $16,6(10,9)$ & $21,2(10,9)$ & $12,8(9,7)$ & $7,8(1,8)$ & $<0,001$ \\
PANSS-Compuesta & $-1,3(8,4)$ & $-2,6(9,9)$ & $1,6(9,6)$ & $0,4(0,9)$ & 0,49 \\
PANSS-General & $32,7(16,7)$ & $39,8(16,1)$ & $29,0(17,2)$ & $18,1(2,9)$ & $<0,001$ \\
SASS & $38,0(11,3)$ & $33,4(10,5)$ & $40,4(7,5)$ & $47,4(8,6)$ & 0,01 \\
OMS-DAS-Total & $6,0(5,7)$ & $8,5(5,5)$ & $3,4(4,8)$ & $1,6(2,2)$ & 0,002 \\
OMS-DAS-Personal & $0,8(1,2)$ & $1,2(1,4)$ & $0,4(0,5)$ & $0(0)$ & 0,007 \\
OMS-DAS-Ocupacional & $1,8(1,8)$ & $2,5(1,8)$ & $1,2(1,8)$ & $0,6(0,8)$ & 0,009 \\
OMS-DAS-Familiar & $1,6(1,5)$ & $2,2(1,5)$ & $0,8(1,1)$ & $0,6(0,7)$ & 0,005 \\
OMS-DAS-Social & $1,9(1,7)$ & $2,6(1,6)$ & $1,0(1,4)$ & $0,8(0,3)$ & 0,001 \\
CSCV-Favorable & $46,1(11,6)$ & $41,6(11,4)$ & $44,8(7,4)$ & $56,7(5,7)$ & $<0,001$ \\
CSCV-Desfavorable & $95,5(33,7)$ & $109,2(34,5)$ & $86,0(23,5)$ & $68,7(12,6)$ & 0,003 \\
\hline
\end{tabular}

Resultados expresados como media (desviación estándar)

* Kruskall-Wallis 
La sintomatología psicótica positiva y negativa presenta una correlación positiva y significativa con la discapacidad, adaptación social y valoración desfavorable de la calidad de vida de los pacientes (Rho $>0.72$ en todos los casos; $\mathrm{p}<0.01$ ). Asimismo, los síntomas psicóticos positivos se correlacionan de forma positiva con los síntomas negativos (Rho $=0.79 ; \mathrm{p}<0.01)$. La adaptación social de los pacientes se correlaciona de forma positiva y significativa con una valoración favorable de su calidad de vida y de manera negativa con la valoración desfavorable de su calidad de vida $($ Rho $=-0.61 ; \mathrm{p}<0.01)$.

Reingresos, variables sociodemográficas (edad y género), sintomatología psicótica y funcionamiento del paciente (adaptación social, discapacidad y calidad de vida) a los cinco años.

En cuanto a los reingresos psiquiátricos como indicador de evolución se obtiene una diferencia significativa $(\mathrm{p}=0.01)$ entre los grupos diagnósticos. El 60\% $(\mathrm{n}=15)$ de los pacientes diagnosticados de psicosis crónica reingresa durante el seguimiento, frente al $20 \%(\mathrm{n}=1)$ de los pacientes con trastorno bipolar y tan sólo el $9.1 \%(n=1)$ de los pacientes que mantienen el diagnóstico de psicosis aguda. Si se analiza el hecho de reingresar, sin considerar el diagnóstico, se observa que estos pacientes presentan una mayor sintomatología psicótica positiva y negativa, así como una menor adaptación social, mayor discapacidad y una valoración más desfavorable de su calidad de vida, todo ello con significación estadística (tabla 5).

Tabla 5.

Reingresos y puntuaciones en las escalas PANSS, OMS-DAS, SASS y CSCV.

\begin{tabular}{|l|c|cc|c|}
\hline Escala & $\begin{array}{c}\text { Global } \\
\text { (n=41) }\end{array}$ & No (24) & Reingresos & (17) \\
\hline PANSS-Positiva & $15,5(11,2)$ & $11,6(8,6)$ & $20,9(12,5)$ & 0,02 \\
PANSS-Negativa & $16,6(10,9)$ & $13,0(9,0)$ & $21,7(11,5)$ & 0,009 \\
PANSS-Compuesta & $-1,3(8,4)$ & $-1,4(7,0)$ & $-1,2(10,4)$ & 0,99 \\
PANSS-General & $32,7(16,7)$ & $24,7(13,6)$ & $43,9(14,2)$ & $<0,001$ \\
SASS & $38,0(11,3)$ & $40,7(9,7)$ & $34,1(12,6)$ & 0,07 \\
OMS-DAS-Total & $6,0(5,7)$ & $3,5(4,6)$ & $9,7(5,0)$ & $<0,001$ \\
OMS-DAS-Personal & $0,8(1,2)$ & $0,4(0,9)$ & $1,4(1,4)$ & 0,005 \\
OMS-DAS-Ocupacional & $1,8(1,8)$ & $1,1(1,6)$ & $2,8(1,6)$ & 0,003 \\
OMS-DAS-Familiar & $1,6(1,5)$ & $0,9(1,2)$ & $2,6(1,3)$ & $<0,001$ \\
OMS-DAS-Social & $1,9(1,7)$ & $1,1(1,5)$ & $2,9(1,3)$ & 0,001 \\
CSCV-Favorable & $46,1(11,6)$ & $48,4(10,6)$ & $42,8(12,5)$ & 0,17 \\
CSCV-Desfavorable & $95,5(33,7)$ & $82,3(29,3)$ & $114,2(31,2)$ & 0,001 \\
\hline
\end{tabular}

Resultados expresados como media (desviación estándar)

* U de Mann-Whitney 
La edad al inicio del episodio psicótico no refleja ninguna correlación con la sintomatología psicótica existente a los cinco años, así como en su adaptación social, discapacidad o calidad de vida (Rho, todos los valores no significativos, $\mathrm{p}>0.05$ ). $\mathrm{Al}$ analizar las diferencias por género, se obtiene que los hombres presentan mayor sintomatología psicótica (positiva y negativa) a los cinco años ( $\mathrm{p}=0.09$ y $\mathrm{p}$ $=0.05$, respectivamente). El nivel de adaptación social es menor en los hombres $(p=0.01)$. El grado de discapacidad es mayor en los hombres que en las mujeres, siendo la diferencia significativa en cuidado personal $(p=0.047)$. Finalmente, las mujeres realizan una valoración más favorable de su calidad de vida y los hombres la describen de forma más desfavorable, aunque en ambos casos sin presentar significación a nivel estadístico (tabla 6).

Tabla 6.

Género y puntuaciones en las escalas PANSS, OMS-DAS, SASS y CSCV.

\begin{tabular}{|l|c|cc|c|}
\hline Escala & $\begin{array}{c}\text { Global } \\
(\mathbf{n = 4 1})\end{array}$ & Hombres (28) & Mujeres (13) & $\mathbf{p}^{*}$ \\
\hline PANSS-Positiva & $15,5(11,2)$ & $17,8(12,6)$ & $10,4(4,8)$ & 0,09 \\
PANSS-Negativa & $16,6(10,9)$ & $19(11,7)$ & $11,5(6,9)$ & 0,05 \\
PANSS-Compuesta & $-1,3(8,4)$ & $-1,4(10,1)$ & $-1,1(2,5)$ & 0,50 \\
PANSS-General & $32,7(16,7)$ & $35,8(17,3)$ & $25,9(13,8)$ & 0,09 \\
SASS & $38,0(11,3)$ & $35,0(11,4)$ & $44,4(8,3)$ & 0,01 \\
OMS-DAS-Total & $6,0(5,7)$ & $7,2(6,0)$ & $3,5(4,0)$ & 0,07 \\
OMS-DAS-Personal & $0,8(1,2)$ & $1,1(1,4)$ & $0,2(0,4)$ & 0,047 \\
OMS-DAS-Ocupacional & $1,8(1,8)$ & $2,1(1,9)$ & $1,2(1,4)$ & 0,12 \\
OMS-DAS-Familiar & $1,6(1,5)$ & $1,9(1,5)$ & $1,0(1,2)$ & 0,09 \\
OMS-DAS-Social & $1,9(1,7)$ & $2,1(1,8)$ & $1,2(1,3)$ & 0.12 \\
CSCV-Favorable & $46,1(11,6)$ & $43,9(12,3)$ & $50,8(8,6)$ & 0,11 \\
CSCV-Desfavorable & $95,5(33,7)$ & $102,2(36,8)$ & $83,2(22,2)$ & 0,17 \\
\hline
\end{tabular}

Resultados expresados como media (desviación estándar)

* U de Mann-Whitney

\section{Discusión.}

Aunque la muestra final es pequeña $(n=41)$, la pérdida es aceptable $(n=7$, $14.5 \%$ ) para tratarse de un estudio de seguimiento a cinco años. En este tipo de trabajos las muestras habitualmente son pequeñas y las pérdidas mayores $(6,14)$.

En el estudio previo, a los dos años, un $43.8 \%$ de los pacientes son diagnosticados de esquizofrenia, esquizoafectivo y bipolar (7). A los cinco años casi el $75 \%$ de los pacientes, inicialmente diagnosticados de psicosis aguda, reciben un diagnóstico de enfermedad psicótica o afectiva crónica. Esta tendencia es recogida en la revisión realizada recientemente por Castagnini y Berrios (15). La mayor estabilidad diagnóstica se observa en los pacientes con trastorno psicótico breve 
y más si el episodio índice se inicia tras la existencia de acontecimientos vitales, en contra de los diagnosticados provisionalmente de trastorno esquizofreniforme. De esta manera, este último diagnóstico no se mantiene en ningún caso a los cinco años, mientras que los que sufrieron un episodio psicótico breve con factores desencadenantes identificables, la mitad no ha evolucionado hacia la cronicidad. En otros estudios también se obtiene una mayor estabilidad para el diagnóstico de psicosis breve, sin bien el período de seguimiento en estos trabajos es menor (1619).

No se aprecian diferencias destacables en la evolución diagnóstica respecto a género o edad de inicio. Las puntuaciones en todas la escalas de la PANNS presentan tres niveles significativos, de mayor a menor, entre los diagnosticados de psicosis crónica, afectivo crónico o psicosis aguda (remitida). Los resultados muestran, de nuevo, tres funcionamientos bien diferenciados. Aquellos que mantienen el diagnóstico original de psicosis aguda muestran a los cinco años una mayor satisfacción con su vida, menor discapacidad funcional y mayor calidad de vida al contrario de los diagnosticados de psicosis crónica, generalmente trastorno esquizofrénico o esquizoafectivo. En una situación intermedia se encuentran aquellos pacientes que han presentado una evolución hacia enfermedades afectivas crónicas como el trastorno bipolar. Estos resultados coinciden con estudios sobre pronóstico y adaptación en pacientes con esquizofrenia, esquizoafectivo y bipolar (20-25).

En el estudio realizado a los dos años se observa ya un $39.6 \%$ de recaídas (7). En este sentido la existencia de recaídas psicóticas, ya observadas durante los dos primeros años, conlleva un cambio en el diagnóstico proporcional al número de ellas (7). Los reingresos a los cinco años son sensiblemente menores que en otros estudios $(14,26)$. En nuestro estudio se valora el reingreso como indicador indirecto de recaída y evolución clínica, obteniéndose una marcada diferencia entre los tres grupos analizados, siendo mayor en los que presentan una psicosis crónica. El reingreso conlleva mayor puntuación en la PANNS, menor satisfacción, mayor discapacidad y menor calidad de vida.

Aunque no se observan diferencias respecto a género en la evolución clínica o diagnóstico a los cinco años, sí que se describe un mejor funcionamiento en las mujeres, de forma que éstas presentan menores puntuaciones en la PANNS, mayor satisfacción personal, menor grado de discapacidad en su actividad y una percepción de calidad de vida más favorable. Podría afirmarse en este sentido, que el diagnóstico a los cinco años es idéntico entre hombres y mujeres, pero el funcionamiento, adaptación y satisfacción con la vida y por lo tanto un pronóstico más favorable es mayor en el sexo femenino (27-30). 
ORIGINALES Y REVISIONES

\section{Conclusiones.}

Se observa que una mayoría de los pacientes evolucionan hacia un diagnóstico psicótico o afectivo crónico, y que es necesario un seguimiento de al menos cinco años, para poder aportar una mayor fiabilidad y estabilidad al diagnóstico realizado.

Entre los criterios diagnósticos utilizados en este estudio (DSM-IV), se puede concluir que un diagnóstico inicial y provisional de trastorno esquizofreniforme parece asegurar a los cinco años un diagnóstico más definitivo de esquizofrenia o esquizoafectivo. Frente a ello, el haber padecido un trastorno psicótico breve y la existencia de factores desencadenantes en el episodio, conlleva un mejor pronóstico y funcionamiento general.

Finalmente, en nuestro estudio las mujeres presentan un funcionamiento y adaptación más favorable que los hombres, aunque padezcan la misma enfermedad.

BIBLIOGRAFIA:

(1) Marneros A, Pillman F, Haring A, Balzuweit S. Acute and transient psychotic disorders. Fortschr Neurol Psychiatr 2000 Apr; 68 Suppl 1: S22-5.

(2) Kampman O, Kiviniemi P, Koivisto E, Väänänen J, Kilkku N, Leinonen E, et al. Patient characteristics and diagnostic discrepancy in first-episode psychosis. Compr Psychiatry 2004; 45: 213-8.

(3) Varma VK, Malhotra S, Yoo ES, Jiloha RC, Finnerty MT, Susser E. Course and outcome of acute non-organic psychotic states in India. Psychiatr Q 1996; 67: 195-207.

(4) Jorgensen P, Bennedsen B, Christensen J, Hyllested A. Acute and transient psychotic disorder: a 1-year follow-up study. Acta Psychiatr Scand 1997; 96: 150-4.

(5) Schwartz JE, Fennig S, Tanenberg-Karant M, Carlson G, Craig T, Galambos N, et al. Congruence of diagnosis 2 years after a first-admission diagnosis of psychosis. Arch Gen Psychiatry 2000; 57: 593-600.

(6) Sajith SG, Chandrasekaran R, Sadanandan Unni KE, Sahai A. Acute polymorphic psychotic disorder: diagnostic stability over 3 years. Acta Psychiatr Scand 2002; 105: 104-9.

(7) Pedrós A, Martí J, Gutierrez G, Tenías JMª Ruescas S. Estabilidad diagnóstica y pronóstico a 2 años de episodios psicóticos agudos. Actas Esp Psiquiatr 2009, 37 (5): 245-51.

(8) Sheehan DV, Lecrubier Y, Harnett-Sheehan K, Janavs J, Weiller E, Bonora LI, et al. Reliability and validity of the MINI International Neuropsychiatric Interview (MINI): according to the SCID-P. European Psychiatry 1997; 12: 232-41.

(9) Kay SR, Fiszbein A, Opler LA. The Positive and Negative Syndrome Scale (PANSS) for Schizophrenia. Schizophr Bull 1987; 13: 261-76.

(10) American Psychiatric Association. Diagnostic and Statistical Manual of Mental Disorders (4th edition) (DSM-IV). Washington, DC: APA. 1994.

(11) WHO Psychiatry Disability Assessment Schedule (WHO/DAS). Genève: WHO, 1988.

(12) Bosc M, Dubini A, Polin V. Development and validation of a social functioning scale, the Social Adaptation Self-evaluation Scale. Eur Neuropsychopharm 1997; 7 (suppl 1): 57-70. 
(13) Giner J, Ibáñez E, Baca E, Bobes J, Leal C, Cervera S. Desarrollo del Cuestionario Sevilla de Calidad de de Vida (CSCV). Actas Luso Esp Neurol Psiquiatr Cienc Afines 1997; 25 (Supl 2): 11-23.

(14) Jäger M, Hintermayr M, Bottlender R, Strauss A, Möller HJ. Course and outcome of firstadmitted patients with acute and transient psychotic disorders (ICD-10: F 23). Eur Arch Psychiatry Clin Neurosci 2003; 225: 209-15.

(15) Castagnini A, Berrios GE. Acute and transient psychotic disorders (ICD-10 F23): a review from a European perspective. Eur Arch Psychiatry Clin Neurosci 2009; 259: 433-43.

(16) Susser E, Fennig S, Jandorf L, Amador X, Bromet E. Epidemiology, diagnosis and course of brief psychoses. Am J Psychiatry 1995; 152: 1743-8.

(17) El Hamoui Y, Yaalaoui S, Moussaoui D, Battas O. Étude de suivi sur deux ans patiens présentant un accès psychotique aigu: modalités évolutives et pronostic. L'Encéphale 2003; 29: 425-9.

(18) Veen ND, Selten JP, Schols D, Laan W, Hoek HW, Van der Tweel I, et al. Diagnostic stability in a Dutch psychosis incidence cohort. Br J Psychiatry 2004, 185: 460-4.

(19) Amini H, Alaghband-rad J, Omid A, Sharifi V, Davari-Ashtiani R, Momeni F, et al. Diagnostic stability in patients with first-episode psychosis. Australas Psychiatry 2005; 13: 388-92.

(20) Menuk M, Legault S, Schmidt P, Remington G. The nosological status of the remitting atypical psychosis. Compr Psychiatry 1989; 30: 53-73.

(21) Jablensky A. Classification of nonschizophrenic psychotic disorders: a historical perspective. Curr Psychiatry Rep 2001; 3: 326-31.

(22) Marneros A, Pillmann F, Haring A, Balzuweit S, Blöink R. The relation of "acute and transient psychotic disorder" (ICD-10 F23) to bipolar schizoaffective disorder. J Psychiatr Res 2002; 36: 16571.

(23) Marneros A, Pillmann F, Haring A, Balzuweit S, R. Blöink R. What is schizophrenic in acute and transient psychotic disorder?. Schizophr Bull 2003; 29: 311-23.

(24) Pilmann F, Marneros A. Longitudinal follow-up in acute and transient psychotic disorders and schizophrenia. Br J Psychiatry 2005; 187: 286-7.

(25) Angst J, Stassen HH, Woggon B. Effect of neuroleptics on positive and negative symptoms and the deficit state. Psychopharmacology (Suppl) 1989; 99: 41-6.

(26) Robinson D, Woerner MG, Alvir JM, Bilder R, Goldman R, Geisler S, et al. Predictors of relapse following response from a first episode of schizophrenia or schizoaffective disorder. Arch Gen Psychiatry 1999; 56: 241-7.

(27) Hafner H, Riecher-Rossler A, Maurer AK, Fatkenheuer B, Loffler W. First onset and early symptomatology of schizophrenia. A chapter of epidemiological and neurobiological research into age and sex differences. Eur Arch Psychaitry Clin Neurosci 1992; 242: 109-18.

(28) Grossman LS, Harrow M, Rosen C, Faull R, Strauss GP. Sex differences in schizophrenia and other psychotic disorders: a 20-year longitudinal study of psychosis and recovery. Compr Psychiatry 2008; 49: 523-9.

(29) Chang WC, Tang JY, Hui CL, Chiu CP, Lam MM, Wong GH, et al. Gender differences in patients presenting with first-episode psychosis in Hong Kong: a three-year follow up study. Aust n Z J Psychiatry 2011; 45 (3): 199-205.

(30) Albert N, Bertelsen M, Thorup A, Petersen L, Jeppesen P, Le Quack P, et al. Predictors of recovery from psychosis Analyses of clinical and social factors associated with recovery among patients with first-episode psychosis after 5 years. Schizophr Res 2011; 125 (2-3): 257-66. 